\title{
El EFeCTO DE los PaQUeTES TECNOLÓGICOS EN LA PRODUCTIVIDAD DEL MAÍZZ EN ECUADOR
}

\author{
Víctor Hugo Sánchez Arizo ${ }^{a}$ y Juan Fernández Sastre ${ }^{b}$
}

Fecha de recepción: 4 de diciembre de 2019. Fecha de aceptación: 11 de junio de 2020.

$$
\text { http://doi.org/10.22201/iiec.20078951e.2020.203.69527 }
$$

Resumen. El presente artículo analiza el efecto de la adopción del maíz híbrido en la productividad por hectárea, utilizando datos de 1622 productores de Ecuador. En particular, se estudia si existen diferencias entre los productores que lo adoptaron por cuenta propia, frente a los que lo hicieron a través de un paquete tecnológico -parcialmente subvencionado por el Estado-, que además de la semilla híbrida incluía tecnologías complementarias, capacitaciones y asistencias técnicas. Los resultados indican que la adopción de semilla híbrida influyó positivamente en la productividad por hectárea, independiente de que ésta fuese adoptada por cuenta propia o a través del paquete tecnológico; aunque el efecto fue mayor sin duda para los productores que adoptaron el paquete tecnológico.

Palabras clave: tecnología agrícola; paquetes tecnológicos; productividad; productores agrícolas; maíz híbrido.

Clasificación JEL: O13; O32; O33; O38; Q16; Q18.

\section{The Effect of Technological Packages on Maize Productivity in Ecuador}

\begin{abstract}
This article analyzes the effect of adopting hybrid maize on productivity per hectare, using data obtained from 1622 producers in Ecuador. Special attention is given to examining whether there are differences between producers who adopted maize independently and those who did so through a technological package -partially subsidized by the state- which, in addition to hybrid seed, included complementary technologies, training, and technical assistance. The findings show that adopting hybrid seed had a positive impact on productivity per hectare, regardless of whether it was adopted independently or as part of a technological package. However, producers who adopted the technological package clearly saw the greatest increase in productivity.

Key Words: agricultural technology; technological packages; productivity; agricultural producers; hybrid maize.

\footnotetext{
a Instituto Nacional de Investigaciones Agropecuarias (INIAP), Ecuador; ${ }^{\mathrm{b}}$ Facultad Latinoamericana de Ciencias Sociales, sede Ecuador. Correos electrónicos: victor.sanchez@iniap.gob.ec y jfernandez@flacso.edu.ec, respectivamente.
} 


\section{INTRODUCCIÓN}

En los países en desarrollo, el sector agrícola está ampliamente caracterizado por la presencia de pequeños productores que utilizan técnicas tradicionales y semillas de menor rendimiento (Lacki, 2011). Esto explica que el índice de productividad, entre la agricultura moderna y la campesina, fuera dos mil veces superior a principios del presente siglo (Carrillo, 2014). De acuerdo con la literatura, en los países en desarrollo, las bajas tasas de adopción se deben a que la nueva tecnología no sólo depende de la disponibilidad de recursos, sino de otros factores relacionados con imperfección de información, el riesgo, la incertidumbre, las restricciones institucionales, el capital humano de los adoptantes, la disponibilidad de insumos y los problemas relacionados con la calidad de las infraestructuras (Foster y Rosenzweig, 1996; Kohli y Singh, 1997).

Ante esta situación, los gobiernos -en su afán de corregir los bajos niveles de productividad agrícola- establecen subvenciones para que los pequeños productores adquieran nuevas variedades de semillas (Todaro y Smith, 2012). En muchos casos, dichas subvenciones se aplican a paquetes tecnológicos que, además de las semillas de mayor rendimiento, incluyen otros insumos (fertilizantes, pesticidas, enraizantes, etcétera); así como también asistencias técnicas sobre cómo producir con nuevas variedades. Esto con el objetivo de proporcionar a los agricultores conocimientos y activos complementarios necesarios para que la adopción de nuevas semillas se traduzca en incrementos de la productividad. Al respecto existe una extensa literatura que destaca que las empresas que adquieren nuevas tecnologías, pero que no invierten en tecnologías complementarias o realizan cambios en la organización de la producción o capacitaciones técnicas, tienen una menor probabilidad de generar ventajas competitivas que aquellas que sí invierten en activos complementarios y formación (Ouadahi, 2008; Boothby et al., 2010). No obstante, pese a la existencia de estos paquetes tecnológicos, persisten productores que todavía utilizan semillas tradicionales; así como también agricultores que adoptan las nuevas variedades sin recurrir a los paquetes tecnológicos.

Ante este contexto, el presente artículo tiene como objetivo analizar si el efecto de la adopción de la semilla de maíz híbrido, sobre la productividad por hectárea, difiere entre los agricultores que la adoptaron por cuenta propia, frente a aquellos que recurrieron a un paquete tecnológico. Cabría esperar que los productores que recurren al paquete tecnológico, que además de la nueva semilla incluye insumos complementarios y capacitaciones técnicas, hagan uso más eficiente $\mathrm{y}$, por tanto, muestren mayor productividad por hectárea. 
En consecuencia, la presente investigación no sólo supone una contribución a la hora de presentar evidencia sobre el efecto de la adopción del maíz híbrido en la productividad por hectárea en el caso de Ecuador, sino además proporciona evidencia sobre si los efectos de la adopción están supeditados a la existencia de conocimientos y tecnologías complementarias proporcionados a través de un paquete tecnológico.

Para ello, se exploraron datos del Ministerio de Agricultura y Ganadería (MAG) correspondientes a los años 2015 y 2016 sobre un total de 1622 productores de maíz duro, localizados en las cuatro principales provincias productoras de maíz de Ecuador: Los Ríos, Guayas, Manabí y Loja. Aunque el maíz duro se produce prácticamente en todo el país, más de $90 \%$ de la superficie cosechada se encuentra en las provincias mencionadas (sIPA, 2020). El caso ecuatoriano resulta idóneo para analizar el efecto de los paquetes tecnológicos agrícolas puesto que durante los años 2015 y 2016 el gobierno subvencionó un paquete tecnológico para la adopción de la semilla de maíz híbrido, que además de la semilla incluía fertilizantes compuestos (nitrógeno, fósforo, potasio), pesticidas, productos fitosanitarios y enraizadores. De manera paralela se brindaba asistencia técnica, capacitación y seguimiento, durante seis meses que iba desde el inicio del cultivo hasta la etapa de cosecha. Dicho paquete fue denominado kit semilla.

Para el cálculo del efecto de la adopción de la semilla híbrida sobre la productividad por hectárea se controla el sesgo de selección. Esto debido a que la adopción de la semilla híbrida, sea por cuenta propia o a través del paquete tecnológico, no fue aleatoria, sino que estuvo supeditada a otras características de los agricultores que también podrían influir en su productividad por hectárea. Se utiliza el método de pareo por propensión (propensity score matching, PSM, por sus siglas en inglés) comparando los resultados de los algoritmos de emparejamiento Kernel y vecino más cercano.

El resto del artículo se organiza de la siguiente manera: la segunda sección revisa la literatura sobre adopción tecnológica y sus efectos, a la vez que muestra la evidencia empírica sobre los efectos de la adopción del maíz híbrido. La tercera sección presenta datos, variables y metodología empleada. En tanto que, la cuarta sección discute las implicaciones de los resultados empíricos. Para finalmente presentar en la quinta sección las conclusiones. 


\section{REVISIÓN DE LA LITERATURA}

En términos generales se pueden distinguir dos corrientes que abordan el problema de la adopción tecnológica (Ruttan, 1996). Por un lado, la corriente ortodoxa considera que la adopción tecnológica está caracterizada por un proceso de elección racional, mediante el cual el productor compara los beneficios derivados de la adopción con sus costes de adquisición. Asume que el productor siempre es capaz de seleccionar la tecnología que más le convenga (Ruttan, 1996). Por otro lado, la corriente evolucionista señala que la adopción tecnológica no debe ser considerada como una elección racional (Selis, 2000), puesto que los agentes toman sus decisiones en un entorno caracterizado por completa incertidumbre. En este sentido, los modelos evolutivos consideran que en el proceso de adopción tecnológica influyen otros factores más allá de los económicos. En consecuencia, para adoptar una nueva tecnología, entre otros factores, también es necesario contar con conocimientos y tecnologías complementarias necesarios para operar eficientemente (Teece, 1986 y 1988). Esto hace que el aprendizaje y el cambio organizacional sea fundamental para que la adopción de nuevas tecnologías genere mayores ganancias de productividad (Boothby et al., 2010). Es decir que en el proceso de adoptar una nueva tecnología influyen no sólo factores económicos, sino también tecnológicos, institucionales e individuales (Foster y Rosenzweig, 1996; Kohli y Singh, 1997).

Las características económicas de los productores influyen en la adopción de nuevas tecnologías, ya que los que cuentan con más recursos, que tienen acceso al crédito y que cuentan con mayores extensiones de tierra serán los más propensos a adoptar nuevas tecnologías (Feder y Umali, 1993). Mientras que, entre los factores tecnológicos, destaca la ventaja relativa de la nueva tecnología frente a la económica (Mwangi y Kariuki, 2015). En este sentido, existe evidencia de que la percepción y el conocimiento de los agricultores sobre las características de una nueva variedad de arroz influyó positivamente en su adopción (Adesina y Zinnah, 1993). Es decir, la experiencia y el conocimiento del agricultor influyen positivamente en la adopción de nuevas tecnologías. Por tanto, un productor más experimentado o con mayor formación puede presentar menor incertidumbre sobre el rendimiento de una nueva tecnología y ser más tendiente a adoptarla sin problema (Rogers, 1971). Otros factores tecnológicos, como la existencia de tecnologías complementarias, también son determinantes para la adopción tecnológica (Teece, 1986).

Los factores institucionales, por su parte, también influyen en la adopción de una nueva tecnología, puesto que el capital social facilita y propicia el in- 
tercambio de información tecnológica (Mignouna et al., 2011). Otro factor institucional destacado es la presencia de agentes de cambio; dado que son los encargados de informar a los productores sobre la existencia y explotación eficiente de las nuevas tecnologías. Los agentes actúan como un enlace entre los productores de la tecnología y sus usuarios. Por tanto, un mayor contacto con estos agentes acelerará el proceso de adopción (Nazziwa-Nviiri et al., 2017). Otras características institucionales son las leyes y regulaciones que influyen en el entorno de mercado; además de las infraestructuras para el transporte y la comunicación (Mwangi y Kariuki, 2015; Nazziwa-Nviiri et al., 2017).

Estudios sobre adopción de tecnologías agrícolas destacan que ciertas características propias del agricultor influyen mucho en su propensión a adoptar nuevas tecnologías. En este sentido, existe evidencia de que la edad del productor influye negativamente en la adopción tecnológica, debido a que los productores de mayor edad tienden a ser más adversos al riesgo (Mauceri et al., 2007; Pan, 2014; Mason y Smale, 2013). El tamaño del hogar también puede afectar a la adopción tecnológica. Por un lado, los hogares de mayor tamaño tienen mayor disponibilidad de fuerza de trabajo, por lo que tenderán a adoptar tecnologías intensivas en trabajo (Tiamiyu et al., 2009; Mauceri et al., 2007) y, por otro, como exponen Saín y Martínez (1999), los hogares con más integrantes utilizan gran parte de sus ingresos para satisfacer sus necesidades básicas, y pueden presentar mayores restricciones al momento de adquirir nuevas tecnologías.

En consecuencia, la adopción de una nueva tecnología agrícola está determinada por multitud de factores, que también condicionan los beneficios que los adoptantes pueden generar a través de su explotación. Es así que el rendimiento de una nueva tecnología depende de que el adoptante posea conocimientos y activos complementarios (Boothby et al., 2010). Los activos complementarios son aquellos requeridos para derivar valor a partir de una inversión en una nueva tecnología; es decir, los productores, para aprovechar el valor de sus inversiones en nueva tecnología necesitan de otras inversiones, conocimientos y activos que complementen su buen funcionamiento (Teece, 1986 y 1988). Por lo tanto, adquirir una nueva tecnología no garantiza buenos rendimientos, ya que algunos productores no son capaces de adaptar su modelo productivo a la misma. En este sentido, existe una amplia literatura empírica que muestra que las empresas que adquieren nuevas tecnologías y simultáneamente invierten en activos complementarios, capacitaciones e implementan cambios organizacionales en el modelo productivo tienden a generar mayores ganancias de productividad que las que no lo hacen (Grander, 2003; Bartel et al., 2007; Boothby et al., 2010). La idea central de dichos trabajos 
es que las ganancias en productividad que resultan de la adopción tecnológica dependen de que se adopten paquetes específicos de nueva maquinaria y equipo, de que se desarrollen cambios organizacionales, así como nuevas habilidades (Boothby et al., 2010).

Aunque los beneficios de adoptar una nueva tecnología agrícola están supeditados a la existencia de conocimientos y activos complementarios o bien a otros factores relacionados con el comportamiento de los precios agrícolas; por lo general la evidencia empírica señala que los adoptantes tienden a estar mejor que los no adoptantes (Besley y Case, 1993; Doss y Morris, 2001; Mendola, 2007; Becerril y Abdulai, 2010). En lo que respecta a los efectos de la adopción del maíz híbrido existen multitud de estudios que, en su mayoría, reportan que la adopción produjo impactos positivos en el bienestar de los agricultores (Kutka, 2011; Olaniyan y Lucas, 2004; Lunduka et al., 2012; Khonje et al., 2015; Karim et al., 2010; Abate et al., 2016; Setimela et al., 2017; Bellon y Hellin, 2011). Por ejemplo, utilizando pareo por propensión, Khonje et al. (2015) mostraron que los adoptantes de la semilla híbrida tenían mayor rendimiento, consumo y seguridad alimentaria que los no adoptantes. Becerril y Abdulai (2010) encontraron que, en los estados mexicanos de Oaxaca y Chiapas, la adopción de maíz híbrido tuvo un impacto positivo en el bienestar de los hogares. También hay evidencia sobre impactos positivos de la adopción del maíz híbrido sobre los beneficios de los productores en Nigeria (Olaniyan y Lucas, 2004). Recientemente, Akhter et al. (2020), también a través de pareo por propensión, presentan evidencia positiva de la adopción de maíz híbrido sobre la producción, el ingreso del hogar y la reducción de la pobreza en Pakistán.

Pese a que existe una extensa literatura empírica sobre los efectos de la adopción del maíz híbrido, ningún estudio analiza sus efectos en Ecuador. Además, tal y como se introdujo, se pretende analizar si su efecto difiere entre los agricultores que lo adoptaron por cuenta propia, frente a aquellos que recurrieron al paquete tecnológico parcialmente subvencionado por el Estado. El interés principal del estudio reside en analizar su impacto en la productividad por hectárea que es una de las medidas de desempeño agrícola más utilizadas (Mason y Smale, 2013; Shively y Ricker-Gilbert, 2013; Tiamiyu et al., 2009). No obstante, la adopción del maíz híbrido podría afectar a otras medidas del bienestar de los productores, independientemente de su efecto en la productividad por hectárea. Por tanto, el hecho de que sólo se presente evidencia respecto a su impacto en la productividad por hectárea hace que no se deba extrapolar dicho efecto a otras medidas del bienestar de los productores o de su entorno. En este sentido, existe evidencia de que el uso de semi- 
llas mejoradas produce efectos negativos tanto para el productor como para el medio ambiente. Desde el punto de vista del productor se observa que puede resultar afectado negativamente por la sustitución de cultivos claves para su nutrición (Shively y Ricker-Gilbert, 2013). Y desde el punto de vista ambiental, se observa que el cultivo de nuevas variedades de maíz puede tener un impacto negativo, derivado del uso de fertilizantes y agroquímicos, de sistemas de riego no sustentables, de la introducción de organismos genéticamente modificados, de la erosión del suelo y la deforestación (Nadal y Wise, 2019; Runge, 2002).

\section{DATOS, VARIABLES Y METODOLOGÍA}

La presente investigación emplea datos proporcionados por la Coordinación General del Sistema de Información Nacional (CGsin) del MAG de Ecuador sobre encuestas realizadas a un total de 1622 productores de maíz duro de las provincias de Los Ríos, Guayas, Manabí y Loja, ${ }^{1}$ correspondiente a los ciclos de siembra de invierno y verano de los años 2015 y 2016 (los datos pueden consultarse en https://bit.ly/2wQgFR3). La base de datos cuenta con información sobre factores económicos (producción, tamaño de finca, superficie cultivada, etcétera); factores tecnológicos (uso de semilla híbrida, uso de fertilizante, uso de maquinaria, tipo de siembra, el sistema de cultivo, el uso de kit tecnológico, etcétera); factores institucionales (pertenencia a una asociación agrícola, posesión de seguro agrícola, etcétera) y factores específicos del productor (edad, número de generaciones que se han dedicado al cultivo del maíz, origen principal de los ingresos, etcétera).

El cuadro 1 muestra una serie de estadísticas descriptivas a nivel provincial, sobre el cultivo del maíz duro. Tal y como se puede observar, el 98\% de la superficie dedicada al cultivo del maíz duro se dá en estas cuatro provincias; sobre todo en las provincias de Los Ríos (45\%) y Manabí (25\%). En media el tamaño de la finca de los productores es bastante pequeño, oscila entre 4.8 ha en Manabí hasta 6.7 ha en Los Ríos. Adicionalmente, se observa que el maíz híbrido está ampliamente difundido en las provincias en estudio. La provincia con menor porcentaje de agricultores utilizando la semilla híbrida es Manabí (65\%); mientras que la provincia con mayor difusión de la misma es Los Ríos,

1 Los Ríos (56 m sobre el nivel del mar, temperatura $25.3^{\circ} \mathrm{C}$, precipitaciones $2060 \mathrm{~mm}$ ). Manabí $\left(18 \mathrm{~m}, 25.7^{\circ} \mathrm{C}, 699 \mathrm{~mm}\right)$. Guayas $\left(120 \mathrm{~m}, 23.7^{\circ} \mathrm{C}, 1181 \mathrm{~mm}\right)$. Loja $\left(200 \mathrm{~m}, 24.9^{\circ} \mathrm{C}, 398 \mathrm{~mm}\right)$ datos consultados en Clima-data.org 
Víctor Hugo Sánchez Arizo y Juan Fernández Sastre

Cuadro 1. Estadísticas descriptivas producción del maíz duro a nivel provincial

\begin{tabular}{lcccccc}
\hline Provincia & $\begin{array}{c}\text { Superficie } \\
\text { cosechada con } \\
\text { maíz respecto al } \\
\text { total del país (\%) }\end{array}$ & $\begin{array}{c}\text { Superficie } \\
\text { de finca } \\
\text { (hectáreas } \\
\text { promedio) }\end{array}$ & $\begin{array}{c}\text { Superficie } \\
\text { cultivada con } \\
\text { maíz (hectáreas } \\
\text { promedio) }\end{array}$ & $\begin{array}{c}\text { Productores } \\
\text { que usan } \\
\text { semilla } \\
\text { hibrida (\%) }\end{array}$ & $\begin{array}{c}\text { Productores } \\
\text { con sistemas } \\
\text { de riego } \\
(\%)\end{array}$ & $\begin{array}{c}\text { Productores que } \\
\text { pertenecen a } \\
\text { una asociación } \\
(\%)\end{array}$ \\
\hline Los Ríos & 45 & 6.7 & 4.9 & 76 & 10 & 43 \\
Manabí & 25 & 4.8 & 3.9 & 65 & 18 & 28 \\
Guayas & 16 & 5.5 & 2.6 & 69 & 27 & 34 \\
Loja & 12 & 6.5 & 3.1 & 70 & 31 & 24 \\
\hline
\end{tabular}

Fuente: encuesta de la CGSIN de los años 2015 y 2016 (MAG, 2018).

donde $76 \%$ de los productores la utilizan. Sin embargo, la difusión de los sistemas de riego es menor en estos lugares. Por ejemplo, en Los Ríos sólo 10\% de los productores del maíz utilizan sistemas de riego; mientras que Loja es la que registra mayor porcentaje (27\%). También se observan algunas diferencias provinciales en términos del porcentaje de productores que pertenecen a una asociación agrícola y que van de $24 \%$ para Loja hasta $43 \%$ en el caso de Los Ríos.

La variable de resultado $(V R)$ sobre la que se pretende estimar el impacto de la semilla híbrida, es la productividad de la tierra expresada en toneladas por hectárea y, de acuerdo a Castro (2016), se calcula a través de la siguiente expresión:

$V R=\frac{p c *(100-\text { porcentaje humedad }- \text { porcentaje impurezas })}{100-(\text { porcentaje humedad fija }- \text { porcentaje impureza fija })}$

Donde:

$p c=\frac{\text { mazorcas por hectárea } * \text { peso promedio de mazorcas }(\text { gramos })}{1000000(\text { gramos } / \text { toneladas métricas })}$

$p c$ : calcula el peso de campo. Es un dato "bruto" que se ajusta considerando la humedad y las impurezas, para obtener la productividad (en toneladas por hectárea).

porcentaje de humedad fija: 13

porcentaje de impureza fija: 1 
mazorcas por hectárea: utilizando la distancia del surco y mazorcas por 10 metros.

peso promedio de mazorca: promedio de los pesos de las mazorcas obtenidas.

porcentaje de humedad e impureza: datos obtenidos en laboratorio de muestras.

Para evaluar el efecto de la semilla híbrida adoptada por cuenta propia, se creó la variable de tratamiento SEMILLA, que es dicotómica y toma valor 1 para los productores que adoptaron la semilla híbrida, pero que la adquirieron por cuenta propia y 0 para los que no utilizan la semilla híbrida. Mientras que la variable de tratamiento que operacionaliza la adopción de la semilla híbrida a través del paquete tecnológico se la denominó KIT, que también es dicotómica y toma valor 1 para los productores que adoptaron la semilla híbrida, a través del paquete tecnológico kit semilla y 0 para los individuos que no utilizan la semilla híbrida. En este punto resulta pertinente aclarar que los productores de maíz duro que no acceden a la semilla híbrida comercial utilizan semilla reciclada de otros híbridos y semilla tradicional. Algunos agricultores en su afán de reducir los costos de la semilla híbrida comercial, utilizan semilla reciclada de sus cosechas pasadas o la adquieren de los vecinos. Se considera que estas semillas son $20 \%$ menos productivas, con alrededor de $40 \%$ menos poder germinativo y mucho más susceptibles al ataque de plagas y enfermedades (Zambrano, 2016). Mientras que la semilla tradicional se emplea en mayor medida en la provincia de Manabí, los materiales tradicionales más conocidos son los denominados "Salprieta", "Criollo" y semillas de la raza "Tusilla” (Yánez, 2014).

En el cuadro 2 se muestra el número de productores tratados y no tratados, para cada tratamiento, así como su media de la productividad por hectárea. Tal y como se puede observar, la mayor parte de los agricultores utilizan la semilla híbrida (1 436 frente a 163) y de ellos la mayoría la adquirió por cuenta propia (981 frente a 455). Finalmente, se puede observar que en media, los productores que utilizan semilla híbrida son más productivos por hectárea y que los que la adoptaron a través del paquete tecnológico son los que muestran mayores niveles de productividad por hectárea. 
Cuadro 2. Estadísticas descriptivas de la productividad de la tierra según las dos variables de tratamiento diseñadas

\begin{tabular}{|c|c|c|c|c|c|}
\hline \multirow[t]{2}{*}{ Tratamiento } & \multirow[t]{2}{*}{ Descripción } & \multirow[t]{2}{*}{ Tratados } & \multirow{2}{*}{$\begin{array}{l}\text { No } \\
\text { tratados }\end{array}$} & \multicolumn{2}{|c|}{ Media y desviación estándar de la VR } \\
\hline & & & & Tratados & No tratados \\
\hline KIT & $\begin{array}{l}\text { Agricultores que adoptaron la semilla } \\
\text { a través del kit semilla }\end{array}$ & 455 & 163 & $\begin{array}{l}5.88 \\
(1.63)\end{array}$ & $\begin{array}{c}4.78 \\
(1.76)\end{array}$ \\
\hline SEMILLA & $\begin{array}{l}\text { Agricultores que adoptaron la semilla } \\
\text { por cuenta propia }\end{array}$ & 981 & 163 & $\begin{array}{l}5.65 \\
(1.86)\end{array}$ & $\begin{array}{c}4.78 \\
(1.76)\end{array}$ \\
\hline
\end{tabular}

Notas: desviación estándar entre paréntesis; $V R=$ variable de resultado.

Fuente: elaboración propia.

\section{Metodología}

Sea $T \in[0,1]$ una de las variables de tratamiento definidas en anteriormente $(K I T$ o $S E M I L L A)$ y $Y \in[0,1]$ la $V R$; el efecto promedio del tratamiento sobre los tratados (ATT, por sus siglas en inglés) se puede calcular de la siguiente manera:

$$
A T T=E\left(Y_{1 i} \mid T=1\right)-E\left(Y_{0 i} \mid T=1\right)
$$

Donde $Y_{1 i}$ es la productividad por hectárea del productor $i$ cuando éste recibe el tratamiento y $Y_{0 i}$ es la productividad por hectárea del mismo productor $i$ si no hubiese recibido el tratamiento. En la ecuación (1) se puede observar el problema metodológico al que se hace frente a la hora de estimar el impacto de la semilla híbrida, pues $E\left(Y_{0 i} \mid T=1\right)$ es un resultado no observable en la base de datos. Por tanto, con la información disponible, el efecto causal sólo puede estimarse por la diferencia de medias entre los individuos tratados y los no tratados, que formalmente se expresa de la siguiente manera:

$$
\left.E\left(Y_{1 i} \mid T=1\right)-E\left(Y_{0 i} \mid \mathrm{T}=0\right)\right)
$$

Nótese que la ecuación (2) permitiría estimar el efecto causal si la asignación al tratamiento hubiese sido aleatoria; dado que en ese caso ocurriría que $\left.E\left(Y_{0 i} \mid T=1\right)=E\left(Y_{0 i} \mid T=0\right)\right)$. Esto último permitiría afirmar que $T$ es independiente de los resultados potenciales; es decir: $T \perp\left(Y_{0 i}, Y_{1 i}\right)$. No obstante, dado que la asignación del tratamiento no es aleatoria, sino que son los productores quienes deciden adoptar la semilla, es 
posible que las diferentes características de los mismos, que influyeron en la adopción del tratamiento, también puedan influir en la $V R$. De tal manera que, con el objetivo de estimar el verdadero efecto causal, esta investigación utiliza el método de pareo por propensión (PSM) (Rosenbaum y Rubin, 1985). El PSM elimina el sesgo de selección construyendo un contrafactual a través de emparejar a los individuos tratados con los no tratados con base en la probabilidad de recibir el tratamiento (Rosenbaum y Rubin, 1985). Así, los individuos tratados son comparados con los no tratados, pero que tienen la misma probabilidad de recibir el tratamiento.

Por tanto, el primer paso metodológico comienza con la estimación para cada individuo (tratado y no tratado) de su probabilidad de recibir el tratamiento con base en una serie de características observables, que se conoce como puntaje por propensión (propensity score). Una vez estimados los puntajes por propensión, para estimar el efecto del tratamiento, se comparan los individuos tratados con los no tratados con un puntaje por propensión similar. Dentro de las estrategias de emparejamiento, un individuo tratado puede ser pareado con el individuo de control con el puntaje por propensión más similar o se puede emparejar al tratado con varios individuos del grupo de control. En este sentido, el algoritmo de emparejamiento vecino más cercano (nearest neighbour matching, NMN, por sus siglas en inglés) consiste en emparejar a cada individuo tratado con aquel no tratado con el puntaje por propensión más próximo (Rodríguez, 2012; Caliendo y Kopeinig, 2005), para posteriormente calcular la diferencia de medias de sus variables de resultado (Becker e Ichino, 2002). Por su parte, el algoritmo de emparejamiento Kernel (Kernel Based Matching, квм, por sus siglas en inglés) calcula el contrafactual usando los promedios ponderados de los individuos en el grupo de control y asigna mayor peso a las observaciones más cercanas en términos de su puntaje por propensión. Este método compara las variables de resultado de tratados y no tratados, asignando mayor peso a estos últimos en función de cuán similares son sus puntajes por propensión (Handouyahia et al., 2013). Por lo tanto, este estimador proporciona algunas ventajas en términos de menor varianza, ya que utiliza más información que otros (Guerzoni y Raiteri, 2015; Caliendo y Kopeinig, 2005).

La validez de este método se basa en el cumplimiento de dos supuestos que se describen a continuación.

Independencia de media condicional: el resultado potencial es independiente, condicionado a la probabilidad de recibir el tratamiento, que se escribe así:

$$
\left.T \perp\left(Y_{0 i}, Y_{1 i}\right) \mid p(x)\right)
$$


donde $p(x)$ es la probabilidad de recibir el tratamiento, condicional a un conjunto de covariables $x$ o puntaje por propensión.

Soporte común: cada individuo tiene una posibilidad de ser tratado y no ser tratado, dadas las covariables, se expresa de la siguiente manera:

$$
0<p(T=1 \mid X=x)<1
$$

Debido al supuesto de independencia de media condicional, en el cálculo del puntaje por propensión, resulta conveniente incluir todas las covariables relacionadas con la $V R$ y con la probabilidad de recibir el tratamiento (Rosenbaum y Rubin, 1985) (véase cuadro 3).

En el cuadro 4 se muestran los resultados de la estimación de los modelos del puntaje por propensión para cada uno de los tratamientos, que dada la naturaleza dicotómica de los mismos, son estimados a través de modelos logit.

Los resultados del cuadro 4 muestran que, independientemente del modo de adopción, los productores con mayor tamaño de finca, más jóvenes, que provienen de más generaciones de productores del maíz y aquéllos cuya principal fuente de ingresos es el maíz fueron más propensos a adoptar el maíz híbrido. Estos resultados están acorde con lo encontrado en otros estudios empíricos sobre determinantes de la adopción de tecnologías agrícolas (Uaiene et al., 2009; Foster y Rosenzweig, 2014; Mason y Smale; 2013; Ali y Abdulai, 2010; Awotide et al., 2016).

Por otro lado, los resultados indican que los productores que pertenencen a una asociación agrícola fueron más propensos a adoptar la nueva semilla a través del paquete tecnológico y que la pertenencia a una asociación no está relacionada con la adopción por cuenta propia. En este sentido, existe evidencia que señala que la pertenencia a una asociación facilita la adopción de nuevas tecnologías agrícolas (Ali y Abdulai, 2010; Uaiene et al., 2009; Tiamiyu et al., 2009), puesto que los productores asociados interaccionan con otros, lo que permite el intercambio de información tecnológica.

Finalmente, los agricultores con cultivo mecanizado fueron más propensos a aquirir la semilla por cuenta propia; mientras que esto no está asociado con la probabilidad de adopción a tavés del kit semilla. 
Cuadro 3. Descripción de variable para el cálculo del puntaje por propensión

\begin{tabular}{|c|c|c|c|c|}
\hline Nombre & Descripción & KIT & SEMILLA & Control \\
\hline Tamaño finca & Logaritmo natural del número de hectáreas & $\begin{array}{l}1.56 \\
(0.79)\end{array}$ & $\begin{array}{l}1.36 \\
(0.94)\end{array}$ & $\begin{array}{l}0.88 \\
(0.86)\end{array}$ \\
\hline Tipo de siembra & $\begin{array}{l}\text { Variable dicotómica que toma valor } 1 \text { para monocultivo } \\
\text { de maíz y } 0 \text { en caso contrario }\end{array}$ & $\begin{array}{l}0.97 \\
(0.18)\end{array}$ & $\begin{array}{l}0.96 \\
(0.19)\end{array}$ & $\begin{array}{c}0.94 \\
(0.24)\end{array}$ \\
\hline Mecaniza siembra & $\begin{array}{l}\text { Variable dicotómica que toma valor } 1 \text { si el productor tiene } \\
\text { siembra mecanizada y } 0 \text { en caso contrario }\end{array}$ & $\begin{array}{c}0.27 \\
(0.45)\end{array}$ & $\begin{array}{c}0.25 \\
(0.43)\end{array}$ & $\begin{array}{c}0.21 \\
(0.41)\end{array}$ \\
\hline Mecaniza cosecha & $\begin{array}{l}\text { Variable dicotómica que toma valor } 1 \text { si el productor tiene } \\
\text { proceso de cosecha mecanizado y } 0 \text { en caso contrario }\end{array}$ & $\begin{array}{c}0.30 \\
(0.46)\end{array}$ & $\begin{array}{c}0.24 \\
(0.43)\end{array}$ & $\begin{array}{c}0.16 \\
(0.37)\end{array}$ \\
\hline $\begin{array}{l}\text { Pertenece a } \\
\text { asociación }\end{array}$ & $\begin{array}{l}\text { Variable dicotómica que toma valor } 1 \text { si el productor } \\
\text { pertenece a una asociación agrícola y } 0 \text { en caso contrario }\end{array}$ & $\begin{array}{l}0.57 \\
(0.50)\end{array}$ & $\begin{array}{c}0.28 \\
(0.45)\end{array}$ & $\begin{array}{c}0.22 \\
(0.42)\end{array}$ \\
\hline Parroquia urbana & $\begin{array}{l}\text { Variable dicotómica que toma valor } 1 \text { si el productor } \\
\text { pertenece a una parroquia urbana y } 0 \text { si pertenece a } \\
\text { una rural }\end{array}$ & $\begin{array}{c}0.64 \\
(0.48)\end{array}$ & $\begin{array}{l}0.64 \\
(0.48)\end{array}$ & $\begin{array}{c}0.58 \\
(0.50)\end{array}$ \\
\hline Edad agricultor & Logaritmo natural de la edad del agricultor & $\begin{array}{l}3.81 \\
(0.30)\end{array}$ & $\begin{array}{c}3.81 \\
(0.32)\end{array}$ & $\begin{array}{l}3.89 \\
(0.35)\end{array}$ \\
\hline Primera generación & $\begin{array}{l}\text { Variable dicotómica que toma valor } 1 \text { si el productor } \\
\text { representa la primera generación dedicada al maíz y } 0 \\
\text { en caso contrario }\end{array}$ & $\begin{array}{c}0.13 \\
(0.34)\end{array}$ & $\begin{array}{c}0.19 \\
(0.40)\end{array}$ & $\begin{array}{c}0.31 \\
(0.46)\end{array}$ \\
\hline Segunda generación & $\begin{array}{l}\text { Variable dicotómica que toma valor } 1 \text { si el productor } \\
\text { representa la segunda generación dedicada al maíz y } 0 \\
\text { en caso contrario }\end{array}$ & $\begin{array}{c}0.48 \\
(0.50)\end{array}$ & $\begin{array}{c}0.40 \\
(0.49)\end{array}$ & $\begin{array}{c}0.34 \\
(0.47)\end{array}$ \\
\hline Más generaciones & $\begin{array}{l}\text { Variable dicotómica que toma valor } 1 \text { si el productor } \\
\text { viene de más de dos generación dedicada al maíz y } 0 \text { en } \\
\text { caso contrario }\end{array}$ & $\begin{array}{c}0.39 \\
(0.49)\end{array}$ & $\begin{array}{c}0.41 \\
(0.49)\end{array}$ & $\begin{array}{l}0.36 \\
(0.48)\end{array}$ \\
\hline Fuente de ingresos & $\begin{array}{l}\text { Variable dicotómica que toma valor } 1 \text { si el máiz } \\
\text { representa la principal fuente de ingresos del productor y } \\
0 \text { en caso contrario }\end{array}$ & $\begin{array}{c}0.86 \\
(0.35)\end{array}$ & $\begin{array}{c}0.81 \\
(0.39)\end{array}$ & $\begin{array}{l}0.67 \\
(0.47)\end{array}$ \\
\hline Año2015_Invierno & $\begin{array}{l}\text { Variable dicotómica que toma valor } 1 \text { si el productor } \\
\text { sembró en el invierno de } 2015 \text { y } 0 \text { en caso contrario }\end{array}$ & $\begin{array}{c}0.47 \\
(0.50)\end{array}$ & $\begin{array}{l}0.15 \\
(0.36)\end{array}$ & $\begin{array}{l}0.26 \\
(0.44)\end{array}$ \\
\hline Año2015_Verano & $\begin{array}{l}\text { Variable dicotómica que toma valor } 1 \text { si el productor } \\
\text { sembró en el verano de } 2015 \text { y } 0 \text { en caso contrario }\end{array}$ & $\begin{array}{c}0.33 \\
(0.47)\end{array}$ & $\begin{array}{c}0.23 \\
(0.42)\end{array}$ & $\begin{array}{l}0.19 \\
(0.39)\end{array}$ \\
\hline Año2016_Invierno & $\begin{array}{l}\text { Variable dicotómica que toma valor } 1 \text { si el productor } \\
\text { sembró en el invierno de } 2016 \text { y } 0 \text { en caso contrario }\end{array}$ & $\begin{array}{c}0.15 \\
(0.35)\end{array}$ & $\begin{array}{l}0.34 \\
(0.47)\end{array}$ & $\begin{array}{c}0.32 \\
(0.47)\end{array}$ \\
\hline Año2016_Verano & $\begin{array}{l}\text { Variable dicotómica que toma valor } 1 \text { si el productor } \\
\text { sembró en el verano de } 2016 \text { y } 0 \text { en caso contrario }\end{array}$ & $\begin{array}{l}0.05 \\
(0.22)\end{array}$ & $\begin{array}{l}0.27 \\
(0.45)\end{array}$ & $\begin{array}{c}0.23 \\
(0.42)\end{array}$ \\
\hline
\end{tabular}

Nota: media y desviación estándar entre paréntesis.

Fuente: elaboración propia. 
Cuadro 4. Estimación del puntaje por propensión para los tratamientos KIT y SEMILLA

\begin{tabular}{|c|c|c|c|c|}
\hline & \multicolumn{2}{|c|}{ KIT } & \multicolumn{2}{|c|}{ SEMILLA } \\
\hline & Coeficiente & Error estándar & Coeficiente & Error estándar \\
\hline Tamaño finca & $0.422 * \star \star *$ & 0.08 & 0.338 *** & 0.058 \\
\hline Tipo de siembra & 0.302 & 0.307 & 0.302 & 0.225 \\
\hline Mecaniza siembra & -0.151 & 0.166 & -0.087 & 0.123 \\
\hline Mecaniza cosecha & 0.278 & 0.163 & 0.338 ** & 0.130 \\
\hline Pertenece a asociación & $0.874^{\star \star \star *}$ & 0.145 & -0.006 & 0.118 \\
\hline Parroquia urbana & 0.110 & 0.135 & 0.001 & 0.103 \\
\hline Edad agricultor & 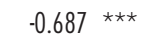 & 0.205 & $-0.496 * * *$ & 0.152 \\
\hline Segunda generación & $0.595 * \star \star$ & 0.174 & $0.359 * \star \star *$ & 0.126 \\
\hline Más generaciones & 0.468 ** & 0.179 & $0.427 * * *$ & 0.129 \\
\hline Fuente de ingresos & 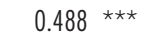 & 0.153 & $0.367 * \star \star *$ & 0.111 \\
\hline \multicolumn{5}{|l|}{ Factores ambientales } \\
\hline Año2015_Invierno & $1.145^{\star \star \star *}$ & 0.224 & -. & -. \\
\hline Año2015_Verano & 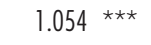 & 0.225 & $0.512 * * *$ & 0.153 \\
\hline Año2016_Invierno & 0.623 ** & 0.242 & $0.446 * \star \star *$ & 0.140 \\
\hline Año2016_Verano & $-\cdot$ & $\cdot \cdot$ & $0.525 * \star \star \star$ & 0.154 \\
\hline Pseudo R2 & 0.290 & & 0.100 & \\
\hline Wald chi2 & 128.080 & & 84.900 & \\
\hline
\end{tabular}

Notas: ${ }^{*} p<0,05 ;{ }^{* \star} p<0,01 ;{ }^{* \star \star} p<0,001$; La variable "Parroquia urbana" se entiende en Ecuador en un sentido de organización territorial; La variable "Primera generación" fue excluida del cuadro porque consituye la categoría de referencia para las otras variables de generaciones; - - dichas variables constituyen la categoría de referencia.

Fuente: elaboración propia.

Antes de poder estimar los efectos de cada tratamiento es necesario comprobar si se cumplen los dos supuestos en los que se basa el método. El supuesto de independencia de media condicional requiere que, tras el emparejamiento, los individuos tratados y no tratados sean iguales en las covariables incluidas en el cálculo del puntaje por propensión y que se definieron en el cuadro 3. En tanto, los cuadros 5 y 6 muestran los resultados del test de diferencia de medias de las covariables entre tratados y no tratados antes y después del emparejamiento, tanto del NNM como del кBM. El cuadro 5 lo hace para el tratamiento KIT mientras que el cuadro 6 lo hace para el tratamiento SEMILLA. 
Cuadro 5. Balance de las covariables luego de aplicado los algoritmos de emparejamiento (NNM y KBM) en el tratamiento KIT

\begin{tabular}{|c|c|c|c|c|c|c|c|c|c|}
\hline & \multicolumn{3}{|c|}{ Antes del emparejamiento } & \multicolumn{3}{|c|}{ Emparejamiento con NNM } & \multicolumn{3}{|c|}{ Emparejamiento con KBM } \\
\hline & Control & Tratado & & Control & Tratado & & Control & Tratado & \\
\hline Tamaño finca & 0.875 & 1.560 & 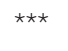 & 1.595 & 1.530 & NS & 1.549 & 1.530 & NS \\
\hline Tipo de siembra & 0.938 & 0.967 & NS & 0.986 & 0.966 & NS & 0.985 & 0.966 & NS \\
\hline Mecaniza siembra & 0.214 & 0.274 & NS & 0.347 & 0.275 & $\star \star$ & 0.332 & 0.275 & NS \\
\hline Mecaniza cosecha & 0.159 & 0.303 & 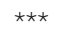 & 0.207 & 0.286 & $\star \star$ & 0.302 & 0.286 & NS \\
\hline Pertenece a asociación & 0.221 & 0.567 & $\star \star \star$ & 0.580 & 0.555 & NS & 0.525 & 0.555 & NS \\
\hline Parroquia urbana & 0.576 & 0.639 & NS & 0.573 & 0.636 & NS & 0.560 & 0.636 & * \\
\hline Edad agricultor & 3.891 & 3.805 & 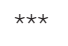 & 3.786 & 3.810 & NS & 3.800 & 3.810 & NS \\
\hline Primera generación & 0.306 & 0.131 & $* \star \star$ & 0.155 & 0.135 & NS & 0.116 & 0.135 & NS \\
\hline Segunda generación & 0.337 & 0.476 & $\star \star$ & 0.395 & 0.471 & $\star \star$ & 0.425 & 0.471 & NS \\
\hline Más generaciones & 0.355 & 0.391 & NS & 0.449 & 0.392 & NS & 0.458 & 0.392 & NS \\
\hline Fuente de ingresos & 0.668 & 0.857 & $\star \star \star \star$ & 0.830 & 0.853 & NS & 0.823 & 0.853 & NS \\
\hline \multicolumn{10}{|l|}{ Factores ambientales } \\
\hline Año2015_Invierno & 0.263 & 0.474 & $\star \star \star$ & 0.435 & 0.460 & NS & 0.478 & 0.460 & NS \\
\hline Año2015_Verano & 0.190 & 0.327 & $* \star \star$ & 0.383 & 0.336 & NS & 0.339 & 0.336 & NS \\
\hline Año2016_Invierno & 0.319 & 0.147 & $\star \star \star *$ & 0.117 & 0.151 & NS & 0.122 & 0.151 & NS \\
\hline Año2016_Verano & 0.226 & 0.050 & 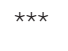 & 0.063 & 0.051 & NS & 0.059 & 0.051 & NS \\
\hline Rango de sesgo (\%) & \multicolumn{3}{|c|}{$7-83.2$} & \multicolumn{3}{|c|}{$0.22-19$} & \multicolumn{3}{|c|}{$0.28-15.7$} \\
\hline
\end{tabular}

$\mathrm{KBM}=$ algoritmo de emparejamiento de Kernel; $\mathrm{NNM}=$ algoritmo de emparejamiento por vecino más cercano; NS= no significativo; ${ }^{\star} p<0,05 ;{ }^{\star \star} p<0,01 ;{ }^{* \star}{ }^{\star \star} p<0,001$.

Fuente: elaboración propia. 
Cuadro 6. Balance de las covariables luego de aplicado los algoritmos de emparejamiento (NNM y KBM) en el tratamiento SEMILLA

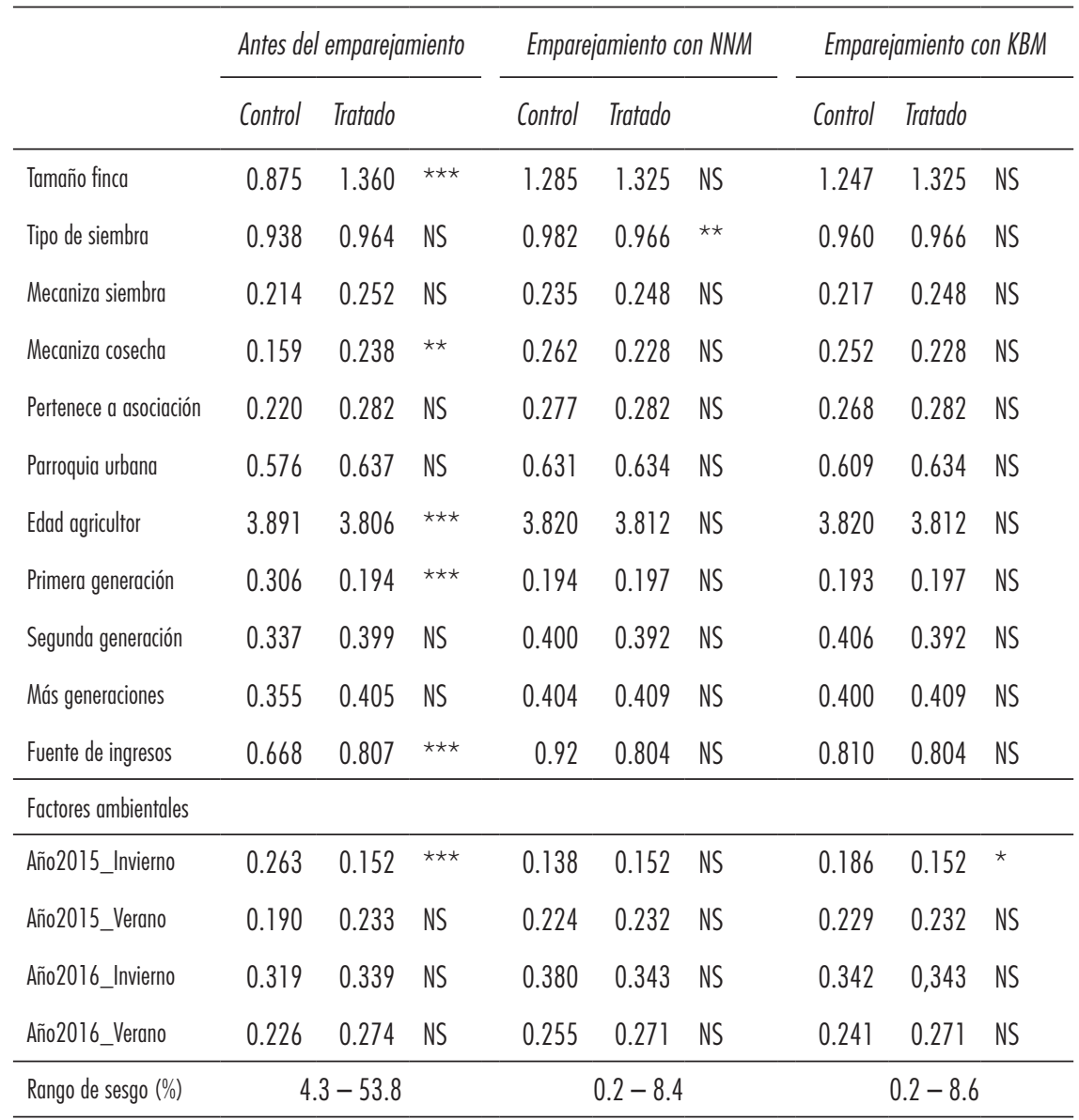

$\mathrm{KBM}=$ algoritmo de emparejamiento de Kernel; $\mathrm{NNM=}$ algoritmo de emparejamiento por vecino más cercano; $\mathrm{NS}=$ no significativo; ${ }^{\star} p<0,05 ;{ }^{* \star} p<0,01 ;{ }^{* \star \star} p<0,001$.

Fuente: elaboración propia.

Los resultados del cuadro 5 señalan que, previo al emparejamiento, los agricultores que adoptaron la semilla a través del kit eran distintos a los no adoptantes, en lo que respecta al tamaño de sus fincas, mecanización de la cosecha, pertenencia a una asociación, edad y número de generaciones dedicadas al cultivo. Sin embargo, tras el emparejamiento las diferencias de medias se reducen, principalmente, con el algoritmo квм que sólo muestra una 
diferencia significativa; el $90 \%$ en lo que respecta a la variable pertenencia a una parroquia urbana. En consecuencia, este algoritmo ofrece resultados más confiables. Por su parte, el cuadro 6 también revela diferencias significativas previas al emparejamiento y que, tras aplicarse, ambos algoritmos consiguen balance entre covariables.

Finalmente, el supuesto de soporte común requiere de la existencia de unidades, tanto en el grupo de control como en el de los tratados, con los mismos puntajes por propensión. Por tanto, debe existir solapamiento en las distribuciones del puntaje por propensión entre ambos grupos. Sin embargo, se darán casos de unidades que no dispongan de una misma probabilidad de recibir el tratamiento, lo que implica que la estimación del efecto se realice en la región de soporte común (Rodríguez, 2012).

La figura 1 muestra las densidades de los valores de propensión para cada uno de los tratamientos, antes y después del emparejamiento квм. ${ }^{2}$ Tal y como se puede observar, las diferencias se corrigen después del procedimiento, las gráficas muestran que la concordancia de los puntajes por propensión reduce las disimilitudes en las distribuciones. El alto grado de superposición señala la buena calidad del procedimiento de coincidencia.

Nótese que el cumplimiento del soporte común provoca la exclusión de los individuos que no disponen de superposición. Cuando la proporción de los individuos dejados fuera de análisis es pequeña, pueden suponerse pocos problemas (Becerril y Abdulai, 2010). En el presente estudio, el cumplimiento del soporte común provocó pocas pérdidas de observaciones: $2.6 \%$ para el caso del tratamiento KIT y $2 \%$ para el SEMILLA (véase cuadro 7). Por lo tanto, se puede desestimar las pérdidas de información durante la lectura de los resultados.

Cuadro 7. Número de individuos por tratamiento en estudio, junto con las pérdidas por el soporte común

\begin{tabular}{lccc}
\hline Tratamiento & $\begin{array}{c}\text { Número de } \\
\text { individuos tratados }\end{array}$ & $\begin{array}{c}\text { Número de individuos } \\
\text { no tratados }\end{array}$ & $\begin{array}{c}\text { Pérdidas para lograr } \\
\text { el soporte común }\end{array}$ \\
\hline KIT & 443 & 163 & $12(2.6 \%)$ \\
SEMILLA & 961 & 163 & $20(2.0 \%)$ \\
\hline
\end{tabular}

Fuente: elaboración propia.

2 La figura correspondiente al algoritmo NNM está disponible solicitándola al autor principal. 
Figura 1. Evaluación de la superposición (algoritmo KBM): a) tratamiento KIT y b) tratamiento SEMILLA a)

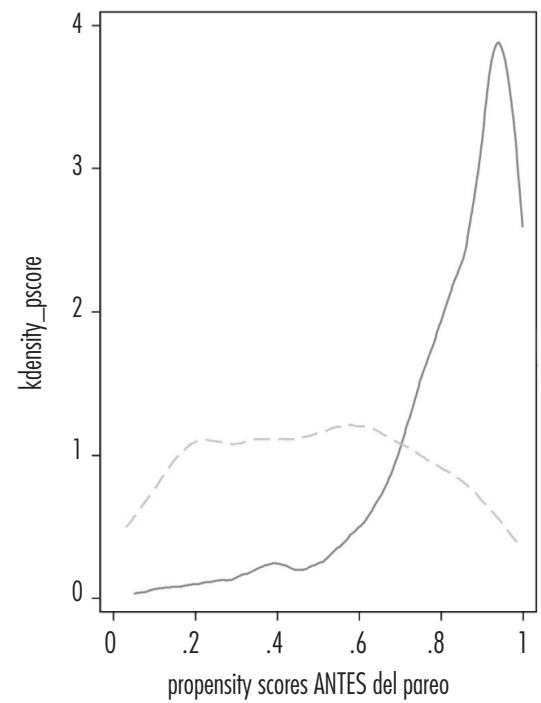

KIT

b)

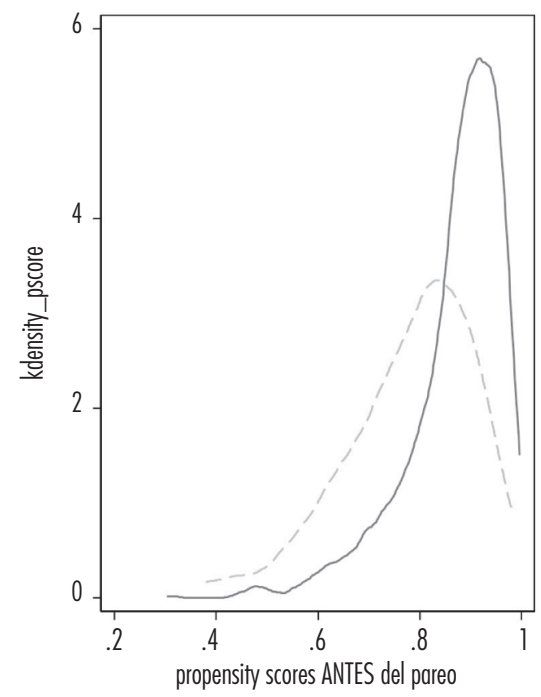

SEMILLA

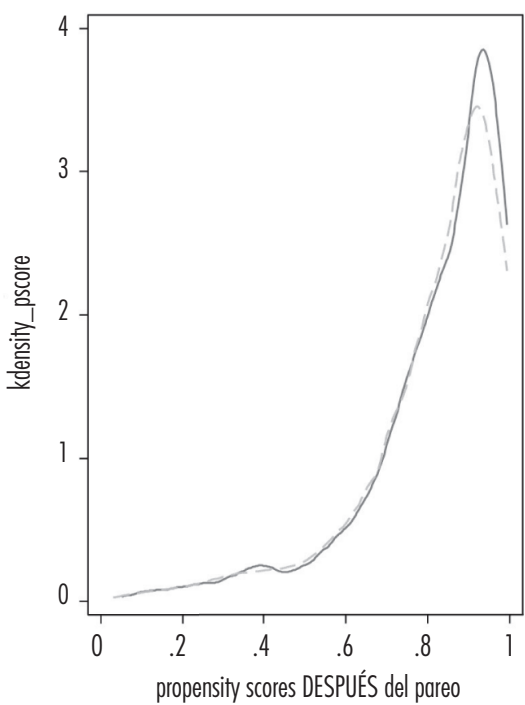

control

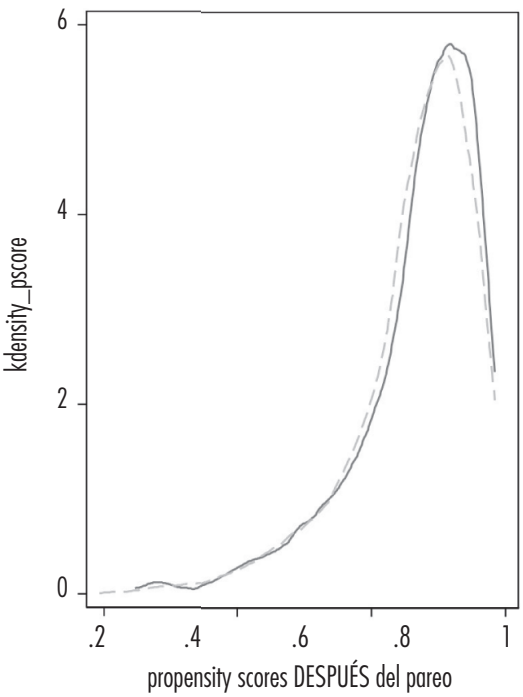

control

Nota: KBM=algoritmo de emparejamiento de Kernel.

Fuente: elaboración propia. 


\section{RESULTADOS}

El cuadro 8 muestra el efecto promedio sobre los tratamientos KIT y SEMI$L L A$ sobre la productividad por hectárea, tanto para el algoritmo de emparejamiento квм como nNM. Tal y como se puede observar, los АтT estimados a través de ambos algoritmos son muy similares, lo que proporciona robustez a los resultados.

Cuadro 8. Efecto promedio del tratamiento sobre los tratados de los tratamientos KIT y SEMILLA

\begin{tabular}{lllcccc}
\hline Algoritmo & Tratamiento & Tratado & Control & ATT & $\begin{array}{c}\text { Error } \\
\text { estándar }\end{array}$ & $\begin{array}{c}\text { Estadístico } \\
\text { " } t^{\prime \prime}\end{array}$ \\
\hline KBM & KIT & 1.734 & 1.461 & $0.272 * * *$ & 0.067 & 4.07 \\
& SEMILLA & 1.671 & 1.549 & $0.122^{* *}$ & 0.042 & 2.88 \\
\multirow{2}{*}{ NNM } & KIT & 1.734 & 1.485 & $0.244 * * *$ & 0.08 & 3.06 \\
& SEMILLA & 1.671 & 1.567 & $0.105 *$ & 0.054 & 1.94 \\
\hline
\end{tabular}

$\mathrm{KBM}=$ algoritmo de emparejamiento de Kernel; $\mathrm{NNM}=$ algoritmo de emparejamiento por vecino más cercano. ${ }^{\star} \mathrm{p}<0,05$; ${ }^{\star \star} p<0,01 ;{ }^{* \star *} p<0,001$.

Fuente: elaboración propia.

Los resultados del cuadro 8 señalan que tanto los productores que adquirieron la semilla híbrida a través del kit semilla como aquellos que lo hicieron por cuenta propia presentan una mayor productividad por hectárea, que los productores que no adquirieron la nueva variedad de semilla y que, además, esto ocurre independientemente del algoritmo de emparejamiento seleccionado. Adicionalmente, los resultados del algoritmo Kernel muestran que los ATT obtenidos para el tratamiento KIT y para el tratamiento SEMILLA fueron de 0.272 y de 0.122 , respectivamente; lo que indica que los productores que adoptaron la semilla junto con las tecnologías complementarias y la capacitación a través del kit semilla obteniendo un mayor incremento en la productividad por hectárea que los que la adoptaron por cuenta propia.

En primer lugar, los resultados están en línea con la mayor parte de la evidencia empírica que señala los efectos positivos en la productividad de los adoptantes de la semilla de maíz híbrido (Besley y Case, 1993; Doss y Morris, 2001; Mendola, 2007; Becerril y Abdulai, 2010; Kutka, 2011; Olaniyan y Lucas, 2004; Lunduka et al., 2012; Khonje et al., 2015; Karim et al., 2010; 
Setimela et al., 2017; Bellon y Hellin, 2011; Akhter et al., 2020). No obstante, además sugieren que el rendimiento de la semilla es mayor cuando se acompaña con tecnologías complementarias y capacitaciones. Esto es así dado que los productores que adquirieron la semilla a través del paquete tecnológico presentan mayor productividad por hectárea que quienes la adquirieron por cuenta propia. Por tanto, estos resultados están en línea con la literatura empírica que señala que los productores que adoptan nuevas tecnologías y simultáneamente adquieren activos complementarios y conocimientos técnicoproductivos, a través de capacitaciones, tienden a generar mayores ganancias de productividad que los que no lo hacen (Grander, 2003; Bartel et al., 2007; Boothby et al., 2010).

Los resultados revelan la importancia de la difusión del maíz híbrido en Ecuador. Sin duda, las diferencias en el rendimiento por hectárea del maíz híbrido y el maíz tradicional explican la amplia difusión del maíz híbrido en estas cuatro provincias de estudio. Adicionalmente, los resultados justifican la idoneidad de complementar las subvenciones a las nuevas variedades de semilla con capacitaciones y tecnologías complementarias para que los productores puedan sacar un mayor rendimiento de las innovaciones agrícolas.

\section{CONCLUSIONES}

El presente estudio analiza el impacto de la adopción del maíz híbrido sobre la productividad por hectárea de los productores de las cuatro principales provincias productoras de maíz duro de Ecuador en los años 2015 y 2016. Adicionalmente, se examina si el impacto de la adopción de la semilla difiere entre los productores que la adquirieron por cuenta propia de los que la adoptaron a través de un paquete tecnológico, que además de la semilla incluía una serie de tecnologías complementarias como fertilizantes, enraizantes, pesticidas y una capacitación sobre el uso de la nueva semilla. Para la estimación de los efectos de la adopción de la semilla híbrida, ya sea por cuenta propia o a través del paquete tecnológico, se utiliza la metodología de pareo por propensión (PSM) a través de los algoritmos de emparejamiento vecino más cercano y Kernel.

Los resultados indican que los productores que adoptaron la semilla híbrida, independientemente del modo de adopción, presentan mayor productividad por hectárea que aquellos que utilizan la semilla tradicional. Por otro lado, se observa, un mayor efecto de la semilla cuando es adoptada a través del paquete tecnológico. Con base en estos resultados se argumenta que el rendimiento que un agricultor puede obtener de una nueva variedad de semilla 
depende de que posea o adquiera tecnologías, conocimientos y habilidades que el uso eficiente de la nueva semilla requiere. Lo que coincide con una extensa literatura que señala que las empresas que adoptan nuevas tecnologías y, simultáneamente, adquieren activos complementarios y conocimientos técnico-productivos generan mayores ganancias de productividad que las que no lo hacen (Grander, 2003; Bartel et al., 2007; Boothby et al., 2010).

Los resultados obtenidos tienen claras implicaciones de cara a la implementación de políticas para la difusión de tecnologías agrícolas en países en desarrollo y emergentes. Esto es así puesto que sugieren que aquellas políticas que faciliten la adquisición de nuevas tecnologías agrícolas a través de subsidios y que, además ofrezcan tecnologías complementarias y capacitaciones, pueden hacer no sólo que los productores sean más propensos a adoptar nuevas tecnologías sino a que hagan un uso más eficiente de las mismas; mejorando así sus niveles de productividad. Es probable que implique la necesidad de coordinar las políticas que proveen incentivos para la adopción de tecnologías con aquellas que proveen de capacitaciones, formación y asistencia técnica. Por tanto, el incremento de la productividad agrícola requiere que los gobiernos implementen un amplio abanico de medidas que van desde incentivar la adopción tecnológica, financiar universidades y centros de investigación para la innovación agrícola y proveer de varios tipos de capacitaciones. En consecuencia, urge el fortalecimiento político-institucional de estos países para que las nuevas tecnologías se difundan con mayor rapidez y los productores obtengan un mayor rendimiento de éstas.

Finalmente, cabe mencionar que a la hora de evaluar los efectos de la adopción del maíz híbrido sería pertinente analizar su impacto en otras medidas de bienestar de los agricultores. Analizar el impacto sólo en términos de productividad por hectárea podría ocultar que la adopción del maíz híbrido podría afectar a otros aspectos. Por tanto, resultaría pertinente extender este estudio a otras variables de resultado y evaluar entonces si el modo de adopción de la nueva semilla también genera impactos diferentes en otros aspectos que son relevantes para los productores agrícolas.

\section{BIBLIOGRAFÍA}

Abate, G., Shahidur T., Borzaga, C. y Getnet, K. (2016), "Rural finance and agricultural technology adoption in Ethiopia: Does the institutional design of lending organizations matter?", Worl Development, vol. 84, DOI <https://doi.org/10.1016/j.worlddev.2016.03.003> 
Adesina, A. y Zinnah, M. (1993), “Technology characteristics, farmers' perceptions and adoption decisions: A Tobit model analysis in Sierra Leone", Agricultural Economics, vol. 9, núm. 4, december, DOI <https://doi. org/10.1016/0169-5150(93)90019-9>

Akhter, A., AbduRahman, B. I. y Dil, A. R. (2020), "Adoption and impact of the maize hybrid on livelihood of the maize growers: Some policy insights from Pakistan", Scientifica, vol. 2020, DOI <https://doi. org/10.1155/2020/5959868>

Ali, A. y Abdulai, A. (2010), "The adoption of genetically modified cotton and poverty reduction in Pakistan", Journal of Agricultural Economics, vol. 61, núm. 1, DOI <https://doi.org/10.1111/j.1477-9552.2009.00227.x>

Awotide, A., Karimov, A. y Diagne, A. (2016), "Agricultural technology adoption, commercialization and smallholder rice farmer's welfare in rural $\mathrm{Ni}$ geria", Agricultural and Food Economics, vol. 4, núm. 3, DOI <https://doi. org/10.1186/s40100-016-0047-8>

Bartel, A., Ichniowski, C. y Shaw, K. (2007), "How does information technology affect productivity? Plant-level comparisons of product innovation, process improvement and worker skills", The Quarterly Journal of Economics, vol. 122, núm. 4, Oxford University Press. Disponible en <www. jstor.org/stable/25098887>

Becerril, J. y Abdulai, A. (2010), "The impact of improved maize varieties on poverty in Mexico: A propensity score-matching approach", World Development, vol. 38, núm. 7, Dor <https://doi.org/10.1016/j. worlddev.2009.11.017>

Becker, S. e Ichino, A. (2002), "Estimation of average treatment effects based on propensity scores", Stata Journal, vol. 2, núm. 4, DOI <https://doi. org/10.1177/1536867X0200200403>

Bellon, M. R. y Hellin, J. (2011), "Planting hybrids, keeping landraces: agricultural modernization and tradition among small scale maize farmers in Chiapas, Mexico", World Development, vol. 8, núm. 39, DOI <https://doi. org/10.1016/j.worlddev.2010.12.010>

Besley, T. y Case, A. (1993), "Modeling technology adoption in developing countries", The American Economic Review, vol. 83, núm. 2, American Economic Association. Disponible en < www.jstor.org/stable/2117697>

Boothby, D., Dufour, A. y Tang, J. (2010), "Technology adoption, training and productivity performance”, Research Policy, vol. 39, núm. 5, DOI <https://doi.org/10.1016/j.respol.2010.02.011>

Caliendo, M. y Kopeinig, S. (2005), "Some practical guidance for the implementation of propensity score matching", Journal of Economic Surveys, vol. 22, núm. 1, DOI <https://doi.org/10.1111/j.1467-6419.2007.00527.x> 
Carrillo, G. (2014), Desarrollo rural y cooperativismo agrario en Ecuador: trayectorias históricas de los pequeños productores en la economía global, Madrid, Ministerio de Agricultura, Alimentación y Medio Ambiente. Disponible en $<$ http://hdl.handle.net/10201/45840>

Doss, C. R. y Morris, M. L. (2001), "How does gender affect the adoption of agricultural innovations? The case of improved maize technology in Ghana", Agricultural Policy, vol. 25, DOI <https://doi.org/10.1016/S01695150(00)00096-7>

Castro, M. (2016), Rendimientos de maiz duro seco en invierno 2016 (Documento de trabajo), Quito, Dirección de Análisis y Procesamiento de la Información. Coordinación General del Sistema de Información Nacional del Ministerio de Agricultura, Ganadería, Acuacultura y Pesca.

Feder, G. y Umali, D. (1993), "The adoption of agricultural innovations a review”, Technological Forecasting and Social Change, vol. 43, núm. 3-4, DOI <https://doi.org/10.1016/0040-1625(93)90053-A>

Foster, A. D. y Rosenzweig, M. R. (1996), "Technical change and humancapital returns and investments: Evidence from the Green Revolution", The American Economic Review, vol. 86, núm. 4, American Economic Association. Disponible en <https://www.jstor.org/stable/2118312>

y Rosenzweig, M. (2014), "Microeconomics of technology adoption", Annual Review of Economics, vol. 2, DoI <https://doi.org/10.1146/ annurev.economics.102308.124433>

Grander, J. P. (2003), "Technology adoption and labour training under uncertainty", Economics of Education Review, vol. 22, DOI <https://doi. org/10.1016/S0272-7757(02)00035-3>

Guerzoni, M. y Raiteri, E. (2015), "Demand-side vs. supply-side technology policies: Hidden treatment and new empirical evidence on the policy mix", Research Policy, vol. 44, DOI <https://doi.org/10.1016/j.respol.2014.10.009>

Handouyahia, A., Haddad, T. y Eaton, F. (2013), "Kernel matching versus inverse probability weighting: A comparative study", International Journal of Mathematical, Computational, Physical, Electrical and Computer Engineering, vol. 7, núm. 8, France, World Academy of Science, Engineering and Technology. Disponible en <https://panel.waset.org/publications/16101/ pdf>

Karim, M. R., Moniruzzaman, M. y Alam, Q. M. (2010), "Economics of hybrid maize production in some selected areas of Bangladesh", Bangladesh Journal of Agricultural Research, vol. 35, núm. 1, DOI < https://doi. org/10.3329/bjar.v35i1.5869> 
Khonje, M., Manda, A., Arega, A. y Kassei, M. (2015), "Analysis of adoption and impacts of improved maize varieties in eastern Zambia", World Development, vol. 66, DOI <https://doi.org/10.1016/j.worlddev.2014.09.008>

Kohli, D. y Singh, N. (1997), The Green Revolution in Punjab, India: The economics of technical change, Technical report, Conference on Agriculture of the Punjab at The Southern Asian Institute, Columbia University.

Kutka, F. (2011), "Open-pollinated vs. hybrid maize cultivars", Sustainability, vol. 3, núm. 4, DOI <10.3390/su3091531>

Lacki, P. (2011), El libro de los pobres rurales. Desarrollo agropecuario: de la independencia al protagonismo del agricultor, Curitiva, RazonEs de SER.

Lunduka, R., Fisher, M. y Snapp, S. (2012), "Could farmer interest in a diversity of seed attributes explain adoption plateaus for modern maize varieties in Malawi?”, Food Policy, vol. 37, núm. 5, DoI <https://doi.org/10.1016/j. foodpol.2012.05.001>

Mason, N. y Smale, M. (2013), "Impacts of subsidized hybrid seed on indicators of economic well-being among smallholder maize growers in Zambia", Agricultural Economics, vol. 44, núm. 6, DOI <https://doi.org/10.1111/ agec.12080>

Mauceri, M., Alwang, J., Norton, G. y Barrera, V. (2007), "Effectiveness of integrated pest management dissemination techniques: a case study of potato farmers in Carchi, Ecuador", Journal of Agricultural and Applied Economics, vol. 39, núm. 3, DOI <https://doi.org/10.1017/S10740708 00023403>

Mendola, M. (2007), "Agricultural technology adoption and poverty reduction: A propensity score analysis for rural Bangladesh", Food Policy, vol. 32, DOI <https://doi.org/10.1016/j.foodpol.2006.07.003>

Mignouna, B., Manyong, M., Rusike, J., Mutabazi, S. y Sekondo, M. (2011), "Determinants of adopting imazapyr resistant maize techology and its impact on household income in western Kenya", AgBioforum, vol. 14, núm. 3, University of Missouri-Columbia. Disponible en <http://agbioforum. org/v14n3/v14n3a07-mignouna.htm>

Ministerio de Agricultura y Ganadería (MAG) (2018), Encuesta de la Coordinación General del Sistema de Información Nacional de los años 2015 y 2016. Recuperado de <https://bit.ly/2wQgFR3>

Mwangi, M. y Kariuki, S. (2015), "Factors determining adoption of new agricultural technology by smallholder farmers in developing countries", Journal of Economics and Sustainable Development, vol. 6, núm. 5, 6, International Institute for Science, Technology \& Education. Disponible en $<$ https://www.iiste.org/Journals/index.php/JEDS/article/view/20710> 
Nadal, A. y Wise, T. (2019), Los costos ambientales de la liberalización agrícola: el comercio de maiz entre México y EE.UU. en el marco del NAFTA, Globalización y medio ambiente: Lecciones desde las Américas (Grupo de Trabajo sobre Desarrollo y Medio Ambiente en las Américas), Heinrich Boll Foundation.

Nazziwa-Nviiri, L., Van Campenhout, B. y Amwonya, D. (2017), Stimulating agricultural technology adoption lessons from fertilizer use among Ugandan potato farmers (Discussion Paper 01608), International Food Policy Research Institute.

Olaniyan, A. B. y Lucas, E. O. (2004), "Maize hybrids cultivation in Nigeriaa review", Journal of Food, Agriculture and Environment, vol. 2, núm. 3 y 4 DOI <https://doi.org/10.1234/4.2004.278>

Ouadahi, J. (2008), "A qualitative analysis of factors associated with user acceptance and rejection of a new workplace information system in the public sector: a conceptual model", Canadian Journal of Administrative Sciences, vol. 25, DOI <https://doi.org/10.1002/cjas.65>

Pan, D. (2014), "The impact of agricultural extension on farmer nutrient management behavior in chinese rice production: A household-level analysis", Sustainability, vol. 10, núm. 6, DOI <https://doi.org/10.3390/su6106644>

Rodríguez, M. (2012), Técnicas de Evaluación de Impacto: Propensity Score Matching y Aplicaciones Prácticas con Stata (Documento No. 2), España, Instituto de Estudios Fiscales.

Rogers, E. (1971), Diffusion of innovations, $3^{\text {rd }}$ edition, New York, Free Press. Rosenbaum, P. y Rubin, D. (1985), "Constructing a control group using multivariate matched sampling methods that incorporate the propensity score", The American Statistician, vol. 39, núm. 1, DOI <10.2307/2683903>

Runge, C. F. (2002), US agricultural policy in 2002: A four dimensional disaster, Winnipeg, George Morris Centre.

Ruttan, V. W. (1996), "What happened to technology adoption diffusion research", Sociologia Ruralis, vol. 36, núm. 1, DOI <https://doi. org/10.1111/j.1467-9523.1996.tb00004.x>

Saín, G. y Martínez, J. (1999), Adoption and use of improved maize by smallscale farmers in southeast Guatemala, Сіммут Economics Paper 99-04, Mexico, Сіммүт.

Selis, D. (2000), "Efectos del cambio tecnológico sobre las condiciones de producción y reproducción del sector hortícola de La Plata”, en R. Ringuelet, Espacio tecnológico, población y reproducción social en el sector hortícola de La Plata, La Plata, UnLP, FAHCE. 
Setimela, P. S., Magorokosho, C., Lunduka, R., Gasura, E., Makumbi, D., Tarekegne, A., Cairns, J. E., Ndhlela, T., Erenstein, O. y Mwangi, W. (2017), "On-farm yield gains with stress-tolerant maize in eastern and Southern Africa", Agronomy Journal, vol. 2, núm. 109, DOI <http://dx.doi. org/10.2134/agronj2015.0540>

Shively, G. y Ricker-Gilbert, J. (2013), Measuring the impact of agricultural input subsidies in sub-Saharan Africa: Evidence from Malawi's farm input subsidy program, Pardue Policy Research Institute (PPRI) Policu Briefs, vol. 1. Disponible en <https://docs.lib.purdue.edu/gpripb/vol1/iss1/4>

Sistema de Información Pública Agropecuaria (SIPA) (2020), Ficha de cultivo de maiz duro seco. Recuperado de <http://sipa.agricultura.gob.ec/index. $\mathrm{php} / \mathrm{maiz}>$

Teece, D. (1986), "Profiting from technological innovation: Implications for integration, collaboration, licensing and public policy", Research Policy, vol. 15, núm. 6, DOI <https://doi.org/10.1016/0048-7333(86)90027-2>

(1988), Profiting from technological innovation: Implications for integration, collaboration, licensing and public policy, London, Printer.

Tiamiyu, S. A., Akintola, J. O. y Rahji, M. A. Y. (2009), "Technology adoption and productivity difference among growers of new rice for Africa in Savanna Zone of Nigeria”, Tropicultura, vol. 27, núm. 4, Belgium, AgriOverseas. Disponible en <http://www.tropicultura.org/text/v27n4/193. pdf>

Todaro, M. y Smith, S. (2012), Economic Development, Boston, AddisonWesley.

Uaiene, R., Arndt, C. y Masters, W. (2009), Determinants of agricultural technology adoption in Mozambique (Discussion papers No. 67E), Mozambique, National Directorate of Studies and Policy Analysis y Ministry of Planning and Development.

Yánez, C. (2014), Programa de Maiz (Informe Anual), Quito, Estación Experimental Santa Catalina del Instituto Nacional de Investigaciones Agropecuarias.

Zambrano, J. (julio 2016), “INIAP, mantiene en vigencia cuatro híbridos para el cultivo de maíz", Revista Técnica Maiz \& Soya. Recuperado de <https:// issuu.com/maizysoya/docs/mys_julio_web> 